\title{
Uma Abordagem para Redução do Tamanho de Shellcodes sem Comprometimento do Comportamento
}

\author{
Geyslan Gregório Bem $^{1}$, Guilherme Álvaro Rodrigues Maia Esmeraldo ${ }^{2}$ \\ ${ }^{1}$ Centro Universitário Internacional (UNINTER) \\ 80020-110 - Curitiba - PR - Brasil \\ ${ }^{2}$ Instituto Federal de Educação, Ciência e Tecnologia do Ceará (IFCE - Campus Crato) \\ 63115-500 - Crato - CE - Brasil \\ geyslan@gmail.com, guilhermealvaro@ifce.edu.br
}

\begin{abstract}
Despite the evolution of different binary attack techniques, Shellcodes are still part of the curricula of courses in the cybersecurity area. The creation of a shellcode may require the use of automated techniques dependent on the scenario and computer architecture, while building it in the smallest size requires a greater effort. This work, which recapitulates the theme, confirming its importance through consultation in the worked issues in courses of the 20 largest Universities in the world, aims to present an approach for optimizing the size of Shellcodes by employing manual techniques, such as PNP, IM , RV. The results show the effectiveness of the proposed approach, which resulting codes have been officially incorporated into the Metasploit project.
\end{abstract}

Resumo. Apesar da evolução das diferentes técnicas de ataque por binários, os Shellcodes continuam fazendo parte dos currículos de cursos na área de cibersegurança. A criação de um shellcode pode demandar o uso de técnicas automatizadas dependentes de cenário e de arquitetura de computador, já construí-lo no menor tamanho exige um esforço maior. Este trabalho, que recapitula o tema, corroborando sua importância por meio de consulta nos conteúdos trabalhados em cursos das 20 maiores Universidades do mundo, tem como objetivo apresentar uma abordagem de otimização do tamanho de Shellcodes, que emprega técnicas manuais, tais como PNP, IM, RV. Os resultados mostram a efetividade da abordagem proposta, cujos códigos resultantes foram incorporados oficialmente ao projeto Metasploit.

\section{Introdução}

Há quase cinco décadas Shellcodes são registrados [Anderson 1972, Spafford 1989] como parte da exploração de vulnerabilidades relacionadas à escrita que extrapola os limites de um buffer de dados. Mas foi a partir da publicação do artigo Smashing The Stack For Fun And Profit [One 1996] que eles passaram a ser conhecidos de forma mais ampla. Segundo [Anley et al. 2007], o termo shellcode foi derivado de seu propósito original - executar um shell. O nome payload, por sua vez, significa um código de propósito genérico, ou o código completo injetado do qual o shellcode faz parte. Atualmente, o uso de ambos os termos é intercambiável sem prejuízo de sentido, haja vista terem se tornado quase sinônimos [Arce 2004]. 
As técnicas de exploração de binários evoluíram em paralelo às tecnologias que prometeram mitigá-las - ESP - Executable-Space Protection, ASLR - Address Space Layout Randomization, Stack Canaries. Tais tecnologias protetivas forçaram a redução do uso prático do shellcode, restringindo-o à exploração de softwares antigos ou que não fazem uso dessas mitigações mais básicas.

No entanto, cursos de universidades continuam mantendo em seus programas conteúdos ou referências relacionados ao aprendizado dessa arte - assim definida por [Foster et al. 2005] -, como etapa preliminar para o aprendizado de técnicas avançadas de exploração ou simplesmente para elucidação dos perigos inerentes à injeção de código. [Cabaj et al. 2018] analisa cursos de Mestrado em Cybersecurity de 21 universidades como pode ser visto na Tabela 1 - nas quais os autores desta pesquisa identificaram, em 12 delas, menções públicas que se relacionam ao conteúdo de shellcode em algum de seus cursos - seja de graduação superior ou de mestrado.

Tabela 1. Cursos [Cabaj et al. 2018] com referência a shellcode

\begin{tabular}{|c|c|c|c|}
\hline Nome & Curso & Termo Encontrado & $\begin{array}{l}\text { Fonte } \\
\end{array}$ \\
\hline $4 \mathrm{TU}$ & $\begin{array}{l}\text { 4TU.CybSec Software } \\
\text { Security (SoS) }\end{array}$ & $\begin{array}{l}\text { buffer and integer } \\
\text { overflows }\end{array}$ & $\begin{array}{l}\text { https://www.4tu.nl/cybsec/en/c } \\
\text { ourse-program/so }\end{array}$ \\
\hline $\begin{array}{l}\text { The Johns Hopkins } \\
\text { University }\end{array}$ & $\begin{array}{l}\text { 695.744: Reverse } \\
\text { Engineering and } \\
\text { Vulnerability Analysis }\end{array}$ & $\begin{array}{l}\text { writing code in } \\
\text { assembly }\end{array}$ & $\begin{array}{l}\text { https://apps.ep.jhu.edu/course } \\
\text { - homepages/3378-695-744-revers } \\
\text { e-engineering-and-vulnerabilit } \\
\text { y-analysis }\end{array}$ \\
\hline $\begin{array}{l}\text { The University of } \\
\text { Southampton }\end{array}$ & $\begin{array}{l}\text { COMP6236: Software } \\
\text { Security }\end{array}$ & $\begin{array}{l}\text { memory violation, } \\
\text { input validation, } \\
\text { privilege escalation }\end{array}$ & $\begin{array}{l}\text { https: } \\
\text { //www. southampton.ac.uk/course } \\
\text { s/modules/comp6236\#syllabus }\end{array}$ \\
\hline $\begin{array}{l}\text { The University of Warwick } \\
\text { in the UK }\end{array}$ & CS263-15: Cyber Security & $\begin{array}{l}\text { reverse engineering, } \\
\text { penetration testing }\end{array}$ & $\begin{array}{l}\text { https://courses.warwick.ac.uk/ } \\
\text { modules/2020/CS263-15.pdf }\end{array}$ \\
\hline $\begin{array}{l}\text { The University of Southern } \\
\text { California }\end{array}$ & $\begin{array}{l}\text { CSCI 430: Introduction to } \\
\text { Computer and Network } \\
\text { Security }\end{array}$ & buffer overflow attacks & http://ccss.usc.edu/430 \\
\hline $\begin{array}{l}\text { Washington University in St. } \\
\text { Louis, USA }\end{array}$ & $\begin{array}{l}\text { CSE637S: Software } \\
\text { Security }\end{array}$ & shellcode & $\begin{array}{l}\text { https://cybersecurity.seas.wus } \\
\text { tl.edu/ning/teaching/S19cse637 } \\
\text { s/lessonPlan.v5 }\end{array}$ \\
\hline Queen's University Belfast & Cyber Range: Red Team & shell code & $\begin{array}{l}\text { https://www.qub.ac.uk/ecit/Abo } \\
\text { utus/Facilities/CyberRange/Fil } \\
\text { etoupload,952093,en.pdf }\end{array}$ \\
\hline Boston University & EC 521: CyberSecurity & payloads & https://ec521.bu.edu \\
\hline $\begin{array}{l}\text { The University of Maryland, } \\
\text { College Park }\end{array}$ & $\begin{array}{l}\text { ENPM6643: Embedded } \\
\text { System Hacking and } \\
\text { Security }\end{array}$ & shellcode & https://mage.umd.edu/enpm 664 \\
\hline George Mason University & $\begin{array}{l}\text { ISA 564: Security } \\
\text { Laboratory }\end{array}$ & shellcode & $\begin{array}{l}\text { https://CS.gmu.edu/media/sylla } \\
\text { bi/Spring2019/ISA_564GreenbergB } \\
\text { 001.pdf }\end{array}$ \\
\hline $\begin{array}{l}\text { Universidad Carlos III de } \\
\text { Madrid }\end{array}$ & $\begin{array}{l}\text { Software systems } \\
\text { exploitation (12386) }\end{array}$ & code injection & $\begin{array}{l}\text { https: } \\
\text { //aplicaciones.uc3m.es/cpa/cpa } \\
\text { /generaFichaPDF } ? \text { ano=2020\&plan= } \\
\text { 325\&asignatura=12386\&idioma }=2\end{array}$ \\
\hline $\begin{array}{l}\text { NYU Tandon School of } \\
\text { Engineering }\end{array}$ & $\begin{array}{l}\text { The Art of Binary } \\
\text { Exploitation: Mobile and } \\
\text { Embedded Systems }\end{array}$ & shellcode & $\begin{array}{l}\text { https://engineering.nyu.edu/si } \\
\text { tes/default/files/2020-09/CS-G } \\
\text { y\%209223\%20MobileEmbeddedExplo } \\
\text { itation.pdf }\end{array}$ \\
\hline
\end{tabular}

O critério de seleção de instituições de nível superior da pesquisa de [Cabaj et al. 2018] não se ateve a uma classificação fixa. Tal fato motivou estes autores a consultarem nas 20 universidades que figuraram no topo da $Q S$ World University Rankings [Symonds 2020] e a consequentemente constatarem haver, em todas as 20 instituições, menção, em seus materiais públicos, a nomes que remetem a shellcodes em pelo menos um dos cursos disponibilizados em cada instituição de ensino - Tabela 2.

Tabela 2. Cursos das 20 melhores universidades [Symonds 2020] que referenciam shellcode

\begin{tabular}{l|l|l|l|l}
\hline \multicolumn{1}{c|}{ Nome } & \multicolumn{1}{c|}{ Curso } & Termo Encontrado & Ano & \multicolumn{1}{c}{ Fonte } \\
\hline $\begin{array}{l}\text { Massachusetts Institute of } \\
\text { Technology (MIT) }\end{array}$ & $\begin{array}{l}\text { 6.858: Computer Systems } \\
\text { Security }\end{array}$ & shellcode & 2020 & $\begin{array}{l}\text { http://css.csail.mit.edu/6.858/ } \\
2020 / \mathrm{abs} / \mathrm{lab} 1 . \mathrm{html}\end{array}$ \\
\hline Stanford University & $\begin{array}{l}\text { CS155: Computer and } \\
\text { Network Security }\end{array}$ & shellcode & 2020 & $\begin{array}{l}\text { https://cs155.stanford.edu/lec } \\
\text { tures/02-ctrl-hijacking.pdf }\end{array}$ \\
\hline \multicolumn{2}{|r}{} & \multicolumn{2}{c}{ Continua na página seguinte } \\
\hline
\end{tabular}




\begin{tabular}{|c|c|c|c|c|}
\hline Nome & $\begin{array}{c}\text { Curso } \\
\end{array}$ & Termo Encontrado & Ano & Fonte \\
\hline Harvard University & $\begin{array}{l}\text { CS 61: Systems } \\
\text { Programming and } \\
\text { Machine Organization }\end{array}$ & buffer overflow & 2020 & $\begin{array}{l}\text { https://cs61.seas.harvard.edu/ } \\
\text { site/2020/Datarep9Activity }\end{array}$ \\
\hline University of Oxford & Digital Systems & buffer overrun & 2020 & $\begin{array}{l}\text { https://spivey.oriel.ox.ac.uk/ } \\
\text { digisys/Lecture_7_-\%E2\%80\%93_Buf } \\
\text { fer_overrun_attacks }\end{array}$ \\
\hline $\begin{array}{l}\text { California Institute of } \\
\text { Technology (Caltech) }\end{array}$ & $\begin{array}{l}\text { CS24: Introduction to } \\
\text { Computing Systems }\end{array}$ & shellcode & 2020 & $\begin{array}{l}\text { https://com.puter.systems/20fa } \\
\text { /projects/03 }\end{array}$ \\
\hline $\begin{array}{l}\text { ETH Zurich - Swiss Federal } \\
\text { Institute of Technology }\end{array}$ & System Security AS20 & buffer overflows & 2020 & $\begin{array}{l}\text { https://syssec.ethz.ch/educati } \\
\text { on/system_security/system_secur } \\
\text { ity_as20.html }\end{array}$ \\
\hline University of Cambridge & Security & shellcode & 2020 & $\begin{array}{l}\text { https://www.cl.cam.ac.uk/teach } \\
\text { ing/1920/Security/security-sli } \\
\text { des-2up.pdf }\end{array}$ \\
\hline UCL & $\begin{array}{l}\text { CS 0133: Distributed } \\
\text { Systems and Security }\end{array}$ & shellcode & 2020 & $\begin{array}{l}\text { http://www0.cs.ucl.ac.uk/staff/ } \\
\text { B.Karp/0133/f2020/lectures/013 } \\
\text { 3-lecture16-exploits.pdf }\end{array}$ \\
\hline Imperial College London & $\begin{array}{l}\text { CO 447: Advanced } \\
\text { Security } 2020\end{array}$ & shellcode & 2020 & $\begin{array}{l}\text { https://co447.doc.ic.ac.uk/sli } \\
\text { des/LEC3.pdf }\end{array}$ \\
\hline University of Chicago & $\begin{array}{l}\text { CMSC 23200/33250: } \\
\text { Introduction to Computer } \\
\text { Security }\end{array}$ & shellcode & 2021 & $\begin{array}{l}\text { https://www.classes.cs.uchicag } \\
\text { o.edu/archive/2021/winter/2320 } \\
\text { 0-1/04.pdf }\end{array}$ \\
\hline $\begin{array}{l}\text { Nanyang Technological } \\
\text { University, Singapore (NTU) }\end{array}$ & $\begin{array}{l}\text { CE/CZ 4062: Computer } \\
\text { Security }\end{array}$ & shell code injection & 2018 & $\begin{array}{l}\text { http://scse.ntu.edu.sg/Programm } \\
\text { es/CurrentStudents/Undergradua } \\
\text { te/Documents/2018/CE/CourseCon } \\
\text { tentCE_Year4.pdf }\end{array}$ \\
\hline $\begin{array}{l}\text { National University of } \\
\text { Singapore (NUS) }\end{array}$ & $\begin{array}{l}\text { CS3235: Computer } \\
\text { Security }\end{array}$ & payload & 2019 & $\begin{array}{l}\text { https: } \\
\text { //www.comp.nus.edu.sg/ hugh/pr } \\
\text { esentations/cs3235/topic10/Lec } \\
\text { t10MachineArchitecture1.pdf }\end{array}$ \\
\hline Princeton University & $\begin{array}{l}\text { COS } 432 \text { : Information } \\
\text { Security }\end{array}$ & shellcode & 2016 & $\begin{array}{l}\text { https://www.cs.princeton.edu/c } \\
\text { ourses/archive/fall16/cos432/h } \\
\text { w2/hw2.pdf }\end{array}$ \\
\hline Cornell University & CS 5430: System Security & shellcode & 2018 & $\begin{array}{l}\text { http://www.cs.cornell.edu/cours } \\
\text { es/cs5430/2018sp/lectures/04-t } \\
\text { hreats/threats.pdf }\end{array}$ \\
\hline University of Pennsylvania & $\begin{array}{l}\text { CIS-331: Introduction to } \\
\text { Networks and Security }\end{array}$ & shellcode & 2019 & $\begin{array}{l}\text { https: } \\
\text { //www.cis.upenn.edu/ sga001/c1 } \\
\text { asses/cis331f19/project1/proj1 }\end{array}$ \\
\hline Tsinghua University & $\begin{array}{l}\text { Security Technologies in } \\
\text { the Cyberspace }\end{array}$ & stack overflow & 2020 & $\begin{array}{l}\text { http://people.iiis.tsinghua.edu } \\
\text {.cn/ wenfei/activities/teachin } \\
\text { g_cybersecurity.html }\end{array}$ \\
\hline Yale University & $\begin{array}{l}\text { CPSC 257: Information } \\
\text { Security in the Real World }\end{array}$ & code injection & 2019 & $\begin{array}{l}\text { https://zoo.cs.yale.edu/classe } \\
\text { s/cs257/lectures/4_Software.pdf }\end{array}$ \\
\hline Columbia University & COMS W4181: Security 1 & shellcode & 2019 & $\begin{array}{l}\text { https: } \\
\text { //www.cs.columbia.edu/ suman/s } \\
\text { ecurity_1/memory_attacks.pdf }\end{array}$ \\
\hline EPFL & $\begin{array}{l}\text { COM-402: Information } \\
\text { security and privacy }\end{array}$ & shellcode & 2020 & $\begin{array}{l}\text { https://com } 402 . e p f l . c h / \text { handout } \\
\text { s/hw1_sol.pdf }\end{array}$ \\
\hline The University of Edinburgh & Secure Programming & shellcode & 2019 & $\begin{array}{l}\text { https://www.inf.ed.ac.uk/teach } \\
\text { ing/courses/sp/2019/labs/lab1/ } \\
\text { Lab1A.pdf }\end{array}$ \\
\hline
\end{tabular}

As ocorrências dos termos encontrados nos cursos da Tabela 2, agrupando-se aproximações e desconsiderando-se repetições, são: shellcode (14), buffer overflow (2), buffer overrun (1), payload (1), stack overflow (1) e code injection (1). As consultas foram realizadas através do Google usando-se o operador avançado site: em conjunto com os termos buscados. Há que se ressaltar que a Tabela 2, contendo apenas um curso por instituição, não exaure a possibilidade de existência de outros cursos nas mesmas instituições referenciadas com conteúdo relacionado.

Ao se identificar facilmente cursos das 20 mais bem avaliadas instituições de ensino superior do mundo tratando do assunto shellcode, percebe-se que esse conhecimento, peça base na exploração de binários [Anley et al. 2007], não foi relegado ao tempo. Considerando-se que há um relevante número de sistemas embarcados - em particular os sistemas profundamente embarcados -, ainda hoje carentes de alguma ou de todas as tecnologias básicas de proteção contra exploração de binários, sejam essas omissões no hardware ou no software [Abbasi et al. 2019, Shackelford 2021] e o fato de o tipo de vulnerabilidade CWE-787 - Out-of-bounds Write - constar na segunda posição da lista Top 25 Most Dangerous Software Weaknesses [MITRE 2020] e em primeiro lugar no relatório Threat landscape for industrial automation systems H2 2019 [Kaspersky ICS CERT 2020], o estudo do tópico shellcode continua pertinente e atual. 
Tendo em vista a importância do tema e que o estudo de técnicas ofensivas de hacking é comumente uma boa maneira para se desenvolver fortes habilidades de ciberdefesa [Joint Task Force on Cybersecurity Education 2018], este trabalho visa contribuir com os estudos de cibersegurança, especificamente de exploração de binários, ao revisitar o tema shellcodes, explicitando meios de refatoração e de redução de payloads existentes no framework Metasploit $(M S F)^{1}$, por este ser um dos frameworks de automação de testes de penetração mais utilizado no mundo [Kennedy et al. 2011]. Para tanto, fazendo uso de três abordagens de construção: (IM) uso de Instruções Menores; (PNP) uso de Propriedades não Padrões; e (RV) Reutilização de valor (código e dados). Essas são técnicas manuais que permitem reduzir o tamanho de um shellcode, dependendo do cenário, e, por consequência, ele se torna melhor pois pode ser alocado em buffers menores e se torna ainda mais útil no caso de aplicações que trazem restrições no tamanho da entrada de dados [Basu et al. 2014].

Cabe explicitar que alguns trabalhos recentes tratam de métodos de confecção de shellcodes para exploração de mitigações avançadas [Sadeghi et al. 2015], de tipos específicos [Németh and Erdődi 2015] e com encoding [Patel et al. 2020]. Os dois primeiros fazem uso de código já existente no próprio binário e o último gera payloads maiores, portanto fogem do escopo desta pesquisa.

O ambiente de análise se restringe ao sistema operacional Linux nas arquiteturas $x 86$ e $x 86-64$. Todavia, com base neste trabalho, a comunidade poderá replicar as demonstrações em outras arquiteturas; salvo respectivas restrições.

\section{Construção do Shellcode}

Sendo uma sequência de instruções de máquina [Perla and Oldani 2010], o shellcode pode ser construído de maneiras análogas a um programa de alto nível. Além dessa variedade de possibilidades de composição, existem técnicas, dependentes da arquitetura e sistema envolvidos, que podem ser utilizadas com o intuito de reduzir ainda mais a quantidade de instruções do código gerado.

Uma etapa comum a se tratar no desenvolvimento do shellcode, é a remoção de $N U L^{2}$ bytes [Anley et al. 2007]. O NUL é considerado um bad char, que, dependendo do binário, pode ser um impeditivo na inserção do bloco de código. Assim como o NUL, outros bytes podem estar na lista de bad chars. Esse aspecto é dependente da implementação do binário em análise. Dessa forma, os autores deste trabalho se limitaram a considerar a existência ou não de NUL bytes nos shellcodes estudados, por conta de sua funcionalidade como delimitador de strings nativo em linguagens como $C$ e Assembly.

Resguardada a efetiva execução das funcionalidades a que se propõe, é senso comum na literatura que um shellcode deve ser construído com a menor quantidade possível de bytes [Hoglund and McGraw 2004, Foster et al. 2005, Anley et al. 2007, Erickson 2008]. Mesmo que a janela de inserção da aplicação explorada possibilite o uso de shellcodes de grande tamanho, há também o fator que permeia todo o processo de exploração de binário: a arte de se construir um payload com o mínimo possível de código garantindo seu funcionamento em variados cenários.

\footnotetext{
${ }^{1}$ Metaspoit. Disponível em: <https://www.metasploit.com/>. Acesso em: 24 jun. 2021.

${ }^{2}$ caractere nulo ' $\backslash 0$ '
} 


\subsection{Instruções Pequenas}

Tem-se buscado utilizar exclusivamente opcodes pequenos - de 1 a 3 bytes na arquitetura $x 86$ e $x 86-64$-, pois é uma das maneiras de se construir um shellcode reduzido. [Foster et al. 2005] afirma que bons desenvolvedores de shellcodes fazem uso dessas instruções com pequenos opcodes.

Há características inerentes à arquitetura x86-64 que possibilitam, com o uso de poucas instruções pequenas, a execução de comportamento pretendido.

\subsection{Propriedades Não Padronizadas}

Os manuais de chamada de sistema ${ }^{3}$ podem conter informações esparsas sobre funcionalidades não mencionadas na sua parte principal; sejam acerca de exceções geradas ou apenas comportamentos deduzidos pelo sistema ao não se seguir a sequência lógica explicitada.

Essas informações, sejam padrões ou não, podem guiar o desenvolvedor acerca de funcionalidades no sistema não claramente documentadas, porém possivelmente úteis para o shellcode em construção. Em casos singulares [Bem 2021a], o código final executa efetivamente, apesar de parecer ter um algoritmo errôneo ou incompleto.

\subsection{Reutilização}

Há outras maneiras de se reduzir o tamanho de um shellcode, sendo duas delas realizadas através da reutilização de instruções injetadas ou da reutilização de dados manipulados nos registradores e na stack [Egypt 2014]. O reuso de instruções se dá através da execução de trechos de código iguais ou similares. Já o reuso de dados se dá pela troca de valores ou endereços nos quais os dados necessários se encontram.

\section{Estudos de Caso}

Esta seção considera dois estudos de caso para demonstrar a abordagem aqui proposta para a redução do tamanho de shellcodes. Os estudos de casos consistem de execve x86 e TCP bind shell x86-64. A seção seguinte apresenta um estudo comparativo entre os shellcodes gerados de forma automatizada com os gerados com o uso da abordagem de redução de tamanho aqui proposta.

\section{1. execve $x 86$}

O propósito inerente no uso de um payload é o de executar código não previsto na confecção original do binário. Para tanto, pode-se utilizar um exemplo simples para a demonstração do uso de um shellcode, que consiste do comumente chamado de execve, o qual, segundo [Foster et al. 2005] é o shellcode mais básico, por conta da chamada de sistema de mesmo nome ${ }^{4}$.

\subsubsection{Metasploit - execve Arbitrário}

Há no $M S F$, numa versão anterior ao produto desta pesquisa, o módulo x $86 /$ exec. rb que constrói um payload destinado a executar, obrigatoriamente, um comando como argumento. $\mathrm{O}$ código gerado faz uso da chamada execve da seguinte forma.

\footnotetext{
${ }^{3} \operatorname{man} 2$ syscall

${ }^{4}$ man 2 execve
} 
Como se observa no payload utilizado no Morris Worm [Spafford 1989] e nos primordiais códigos do UNIX [Spinellis 2017], a chamada de sistema execve executava efetivamente sem definir a lista de argumentos, fazendo uso de um comportamento não previsto nos respectivos manuais.

Essa característica é corroborada numa das primeiras versões do kernel do Linux, v0.01 [Torvalds 1991] - a mais antiga disponível com versionamento -, assim como em uma das mais atuais, v5.11 [Biederman 2020]. Sem prejuízo da execução, o kernel Linux atribui à argc o valor 0 no caso de argv ser NULL. ${ }^{7}$

No manual da chamada de sistema execve do atual Linux [Kerrisk 2021], assim como no do primeiro UNIX [Spinellis 2017], consta que, por convenção, a primeira string argv [0 ] deve conter o nome do arquivo a ser executado. Contudo, no manual do Linux foi adicionada [Kerrisk 2007] informação na seção Notas, ao lado de ressalvas sobre possível incompatibilidade com outros sistemas UNIX, afirmando que argv e envp podem ser definidos como NULL.

Com o propósito principal de se poder obter um payload mínimo para execução de um shell, algumas mudanças no gerador $\times 86 /$ exec.rb foram propostas. A submissão de código envolveu novo comportamento, nova opção e aspectos de refatoração [Bem 2021b].

O novo comportamento se resumiu a possibilitar que o CMD não seja definido, chamando execve sem argumentos. O shellcode gerado, com base nessa PNP, atinge seu propósito, livre de NUL bytes, com apenas 21 bytes.

Código 2. MSF payload - execve("/bin//sh", NULL, NULL)

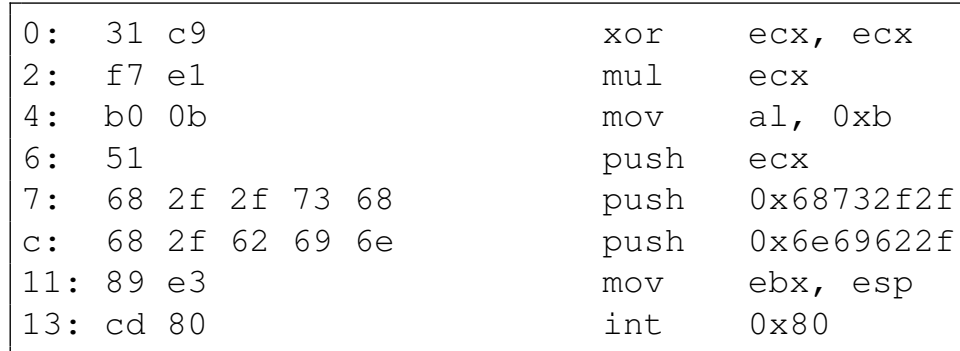

\subsubsection{Otimização do Tamanho do execve symlink}

Os execve a seguir são produtos deste trabalho que fazem uso de IM, contudo não foram submetidas ao $M S F$ pois dependem de características singulares do ambiente explorado.

Em um ambiente de exploração que há possibilidade de escrita no CWD, Current Working Directory, do binário, pode-se reduzir ainda mais o payload execve, substituindo as duas instruções push (10 bytes), que inserem a string "/bin//sh" na stack, por um único push 0x73 ( 2 bytes), inserindo apenas "s". Para tanto, s deve ser um link simbólico para o binário / bin/sh. A nova versão inclui apenas 13 bytes.

Código 3. execve("s", NULL, NULL)

$\frac{0: 31 \text { c9 }}{{ }^{7}((\operatorname{void} *) 0)}$ xor ecx, ecx


No cenário em que não se tem permissões de acesso de escrita no CWD, uma solução é por o segundo estágio no / tmp, se este path estiver montado com permissões de execução. Por ser necessário utilizar o caminho absoluto $/ \mathrm{tmp} / \mathrm{s}$, tal procedimento resulta em um shellcode com tamanho de 20 bytes.

Código 4. execve("/tmp/s", NULL, NULL)

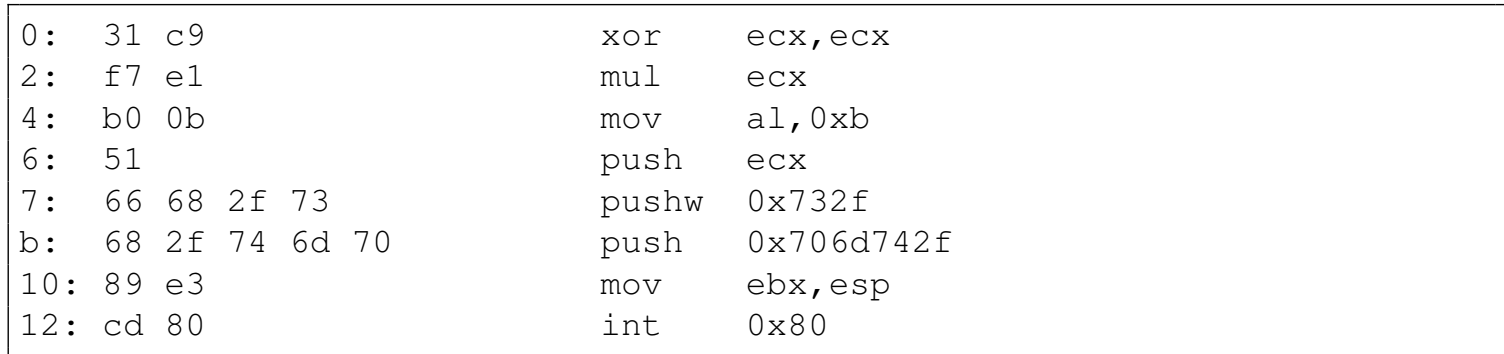

\subsubsection{Ressalvas Acerca dos Privilégios}

Apesar dos seus tamanhos reduzidos, os shellcodes execve demonstrados não mantêm os privilégios do binário explorado pois o bash testa se está os mesmos estão executando com o bit setuid setado [Fox and Ramey 2021]. Para tentar evitar tal comportamento, pode-se usar uma das versões reduzidas com o intuito de executar um segundo estágio responsável por usar a opção - p (privileged) que é checada em conjunto com o setuid.

O segundo estágio deve compor e chamar execve da seguinte maneira.

Código 5. Segundo Estágio - execve("/bin/sh", ["/bin/sh", "-p"], NULL)

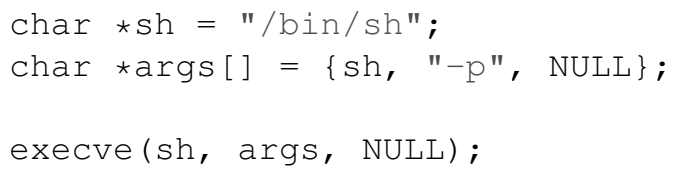

É pertinente registrar que binários suid, que reduzem privilégios com o intuito de deixar a execução segura, podem ter seus $i d s$ restaurados através de chamada de sistema ${ }^{8}$ [Kerrisk 2010] a ser posicionada antes da chamada do execve.

\subsection{TCP bind shell $x 86-64$}

Para [Foster et al. 2005] o shellcode do tipo port-binding é um dos mais comuns para exploração de vulnerabilidades remotas. Seu funcionamento se resume em aguardar uma conexão numa porta de rede e, após aceita, vincular um shell a essa conexão [Foster et al. 2005, Erickson 2008], portanto, também é conhecido como shell-binding.

\footnotetext{
${ }^{8}$ man 2 setuid setreuid setresuid
} 
Para esse propósito, o payload, ilustrado na Figura 1, executa uma série de chamadas de sistema: socket, bind, listen etc.

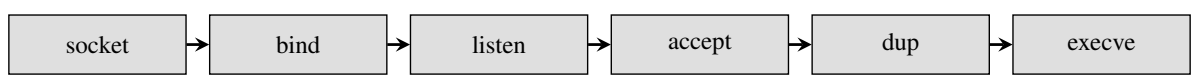

Figura 1. Chamadas de sistema de um shell-binding convencional

\subsubsection{Metasploit - TCP bind shell}

O módulo x64 / shel1_bind_tcp.rb, disponível no MSF [ricky 2021], gera um payload com a sequência lógica da Figura 1 e tamanho final de 86 bytes, contendo 2 ou 3 NUL bytes, a depender do número da porta escolhida através da opção LPORT ${ }^{9}$. Sua versão livre de NUL bytes é gerada com 136 bytes, após encoding.

\subsubsection{Portas dinâmicas}

Assim como no execve sem argv, uma chamada de função usada no shell-binding possui comportamento incomum que facilita a construção com foco na redução de código.

Um bind implícito [Kerrisk 2010, Kerrisk 2014] ocorre ao se chamar listen para um socket TCP que não possui um endereço vinculado. O kernel atribui ao socket uma porta dinâmica (efêmera) disponível a ser descoberta através do uso de um port scanner, tal como o nmap, tornando possível remover o código relacionado ao b ind do shellcode.

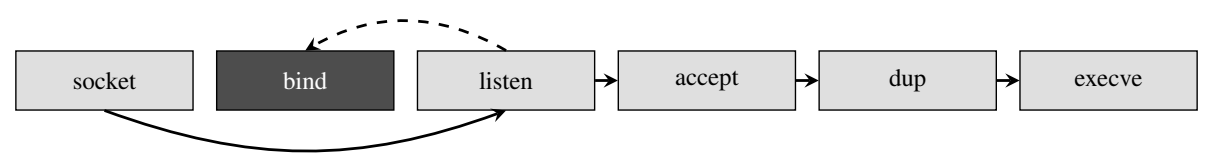

Figura 2. Chamadas de sistema de um shell-binding implícito

Uma versão prévia que segue a lógica da Figura 2, livre de NUL bytes e com apenas 57 bytes, já se encontrava incorporada ao MSF com nome x64/shell_bind_tcp_random_port.rb [Bem 2018]. Nessa versão reduzida, utilizou-se a PNP do bind implícito e RV, a exemplo da instrução de máquina xchg. Com ela foi possível reutilizar valores constantes em registradores diferentes.

Código 6. MSF payload - trecho do shell_bind_tcp_random_port

1d: $4897 \quad x c h g$ rdi, $\operatorname{rax}$

Quando construído para 64 bits o shellcode pode fazer uso de algumas instruções, como a xchg do Código 6, porém com os operandos legados da arquitetura 32 bits, registradores edi e eax, diminuindo, neste contexto, um byte no tamanho do opcode e ainda zerando a parte superior dos registradores envolvidos - zero-extension [AMD 2020]. Essa

\footnotetext{
${ }^{9}$ set LPORT number
} 
técnica também pode ser útil para obter o valor nulo em algum registrador ou trocar valores entre registradores com um custo de opcodes menor. Haja vista o payload previamente incorporado não fazer o efetivo uso das menores instruções disponíveis, sua refatoração se tornou mais um produto desta pesquisa.

\section{Resultados}

Os métodos utilizados na redução do execve foram o de propriedade não padrão (PNP) e o de instrução menor (IM). Ambos proporcionaram payloads com tamanhos no mínimo $70 \%$ menores que o do payload sem NUL bytes gerado pelo framework. A versão execve sem argv foi incorporada ao repositório do MSF [Bem 2021b].

Tabela 3. Redução do payload execve que executa um shell - x86

\begin{tabular}{|c|c|c|c|c|}
\hline Versão & Bytes & Redução $\approx$ & Método & Legenda \\
\hline arbitrário & 43 & - & - & \multirow{5}{*}{$\begin{array}{l}1 \text { - Do MSF, usado como base p/redução } \\
2 \text { - Payload incorporado ao MSF }\end{array}$} \\
\hline arbitrário $s / N U L^{I}$ & 70 & - & - & \\
\hline sem $\operatorname{argv}^{2}$ & 21 & $70 \%$ & PNP & \\
\hline symlink CWD & 13 & $81 \%$ & PNP, IM & \\
\hline symlink /tmp & 20 & $71 \%$ & PNP, IM & \\
\hline${ }^{2}$ Commit: 3422387 & & & & \\
\hline
\end{tabular}

Na refatoração do shell-binding de porta dinâmica [Bem 2018], com o uso da xchg, foi possível engendrar uma melhor reutilização de valores contidos nos registradores entre as chamadas de sistema. Durante o período desta pesquisa foram criadas e incorporadas oficialmente ao $M S F$ três versões desse shellcode, ficando o resultado final com tamanho de 51 bytes [Bem 2021a].

Tabela 4. Redução do payload shell-binding - x86-64

\begin{tabular}{|c|c|c|c|c|}
\hline Versão & Bytes & Redução $\approx$ & Método & Legenda \\
\hline porta arbitrária & 86 & - & - & \multirow{6}{*}{$\begin{array}{l}1 \text { - Do MSF, usado como base p/redução } \\
2 \text { - Payload incorporado ao MSF } \\
\text { PNP - Propriedade Não Padrão } \\
\text { IM - Instrução Menor } \\
\text { RV - Reutilização de Valor }\end{array}$} \\
\hline porta arbitrária $s / N U L^{I}$ & 136 & - & - & \\
\hline porta dinâmica v1 & 57 & $58 \%$ & PNP, RV & \\
\hline porta dinâmica v $2^{2}$ & 53 & $61 \%$ & PNP, IM, RV & \\
\hline porta dinâmica v3 $3^{2}$ & 52 & $61 \%$ & PNP, IM, RV & \\
\hline porta dinâmica $v 4^{2}$ & 51 & $62 \%$ & PNP, IM, RV & \\
\hline${ }^{2}$ Commits: 5edb4cd, ab307fb e 74a77fb. & & & & \\
\hline
\end{tabular}

Todos os métodos manuais aplicados geraram shellcodes reduzidos com todas suas propriedades preservadas, com exceção das versões symlink do execve, como explicitado na Subsubseção 3.1.2. O uso de PNP se destaca pela possibilidade de aplicação em todas as reduções apresentadas, sendo que obteve-se o resultado de $70 \%$ pela aplicação isolada desse método.

A arquiteturas x86 e x86-64 e sistema operacional Linux foram propriedades necessárias para tais resultados, uma vez que, a exemplo, alguma outra arquitetura pode impossibilitar o uso de IM. 


\section{Considerações Finais}

A depender das necessidades de execução e do ambiente em questão, construir um pequeno artefato de código com o mínimo de bytes possível se renova como uma arte a ser exercida e pesquisada.

Com o intuito de contribuir para a comunidade de cibersegurança, em especial a que trata de exploração de binários, foram apresentadas neste trabalho idiossincrasias de chamadas do sistema operacional Linux usadas como base para a confecção de shellcodes da arquitetura x86 e x86-64 com tamanho reduzido. Esta pesquisa gerou produtos de código que foram submetidos, aceitos e incorporados ao framework Metasploit, assim como outros meramente explanatórios - Código 3 e Código 4. Em trabalhos futuros, pretende-se aplicar os métodos desta pesquisa nos demais módulos do referido framework.

\section{Agradecimentos}

Os autores são gratos a Pedro Fausto Rodrigues Leite Junior pela prontidão nas várias revisões que retornaram sempre com dicas preciosas, e a Tiago de Bem Natel de Moura, partícipe de discussões que fomentaram a base desta pesquisa.

\section{Referências}

Abbasi, A., Wetzels, J., Holz, T., and Etalle, S. (2019). Challenges in Designing Exploit Mitigations for Deeply Embedded Systems. In 2019 IEEE European Symposium on Security and Privacy (EuroS\&P), pages 31-46.

AMD (2020). AMD64 Architecture Programmer's Manual, Volume 1: Application Programming. Advanced Micro Devices, publication 24592, revision 3.23.

Anderson, J. P. (1972). Computer Security Technology Planning Study. Volume 2. Technical report, Anderson (James P) and Co Fort Washington PA.

Anley, C., Heasman, J., Linder, F., and Richarte, G. (2007). The Shellcoder's Handbook: Discovering and Exploiting Security Holes. John Wiley \& Sons, Inc., USA, 2nd edition.

Arce, I. (2004). The Shellcode Generation. IEEE Security and Privacy, 2(5):72-76.

Basu, A., Mathuria, A., and Chowdary, N. (2014). Automatic generation of compact alphanumeric shellcodes for x86. In Prakash, A. and Shyamasundar, R., editors, Information Systems Security, pages 399-410, Cham. Springer International Publishing.

Bem, G. G. (2018). Linux Command Shell, Bind TCP Random Port Inline. In Metasploit 6.0.26. Github. https://github.com/rapid7/metasploit-framework/blo b/6.0.26/modules/payloads/singles/linux/x64/shell_bind_tcp_ran dom_port.rb. Acessado: 05/04/2021.

Bem, G. G. (2021a). Linux Command Shell, Bind TCP Random Port Inline. In Metasploit 6.0.37. Github. https://github.com/rapid7/metasploit-framework/blo b/6.0.37/modules/payloads/singles/linux/x64/shell_bind_tcp_ran dom_port.rb. Acessado: 29/01/2021.

Bem, G. G. (2021b). payload/x86/exec.rb - refactoring, metasm, new NullFreeVersion option. In Metasploit. Github. https://github.com/rapid7/metasploit-fra mework/pull/14661. Acessado: 28/03/2021. 
Biederman, E. W. (2020). exec: Factor bprm_stack_limits out of prepare_arg_pages. In Linux Kernel. Kernel. https://git.kernel.org/pub/scm/linux/kernel/gi t/torvalds/linux.git/commit/fs/exec.c?h=v5.11\&id=d8b9cd549ecf 0 f $3 \mathrm{dc} 8 \mathrm{da} 42 \mathrm{ada} 5 \mathrm{f} 0 \mathrm{ce} 73 \mathrm{e} 8 \mathrm{ed} 5 \mathrm{f} 1 \mathrm{e}$. Acessado: 28/03/2021.

Cabaj, K., Domingos, D., Kotulski, Z., and Respício, A. (2018). Cybersecurity education: Evolution of the discipline and analysis of master programs. Computers \& Security, 75:24-35.

Egypt (2014). Shellcode Golf: Every Byte is Sacred. https ://www.rapid7.com/b log/post/2014/02/14/shellcode-golf. Acessado: 06/09/2021.

Erickson, J. (2008). Hacking: The Art of Exploitation. No Starch Press, USA, 2nd edition.

Foster, J. C., Osipov, V., Bhalla, N., Heinen, N., and Aitel, D. (2005). Buffer Overflow Attacks. Syngress, Burlington.

Fox, B. and Ramey, C. (2021). shell.c. In GNU Bash, the Bourne Again SHell. Savannah. http://git.savannah.gnu.org/cgit/bash.git/tree/shell.c?h=bash5.1\#n505. Acessado: 29/03/2021.

Hoglund, G. and McGraw, G. R. (2004). Exploiting Software: How to Break Code. Addison-Wesley Professional, USA.

Joint Task Force on Cybersecurity Education (2018). Cybersecurity Curricula 2017: Curriculum Guidelines for Post-Secondary Degree Programs in Cybersecurity. Association for Computing Machinery, New York, NY, USA.

Kaspersky ICS CERT (2020). Threat landscape for industrial automation systems. H2 2019. Kaspersky. https://ics-cert.kaspersky.com/media/KASPERSKY_H 22019_ICS_REPORT_FINAL_EN.pdf. Acessado: 26/03/2021.

Kennedy, D., O'Gorman, J., Kearns, D., and Aharoni, M. (2011). Metasploit: The Penetration Tester's Guide. No Starch Press, USA, 1st edition.

Kerrisk, M. (2007). Add text noting that Linux allows 'argv' and 'envp' to be NULL. In Linux Kernel. Kernel. https://git.kernel.org/pub/scm/docs/man-pages/ man-pages.git/commit/man2/execve.2?id=456960740c5b50c3a6alc953 9fd4d8851e2eb885. Acessado: 28/03/2021.

Kerrisk, M. (2010). The Linux Programming Interface: A Linux and UNIX System Programming Handbook. No Starch Press, USA, 1 edition.

Kerrisk, M. (2014). ip.7: Note cases where an ephemeral port is used. In Linux Kernel. Kernel. https://git.kernel.org/pub/scm/docs/man-pages/man-pages . git/commit/man7/ip.7?id=509c1c26f0e0d02c9f128bf2b8df5923c4634d e1. Acessado: 06/04/2021.

Kerrisk, M. (2021). execve(2) - Linux manual page. man7. https://man7.org/lin ux/man-pages/man2/execve.2.html. Acessado: 02/04/2021.

MITRE (2020). CWE Top 25 Most Dangerous Software Weaknesses. MITRE. https: //cwe.mitre.org/top25/archive/2020/2020_cwe_top25.html. Acessado: 24/03/2021. 
Németh, Z. L. and Erdődi, L. (2015). When every byte counts - Writing minimal length shellcodes. In 2015 IEEE 13th International Symposium on Intelligent Systems and Informatics (SISY), pages 269-274.

One, A. (1996). Smashing The Stack For Fun And Profit. Phrack Magazine, 7(49).

Patel, D., Basu, A., and Mathuria, A. (2020). Automatic Generation of Compact Printable Shellcodes for x86. In 14th USENIX Workshop on Offensive Technologies (WOOT 20). USENIX Association.

Perla, E. and Oldani, M. (2010). A Guide to Kernel Exploitation: Attacking the Core. Syngress Publishing.

ricky (2021). Linux command shell, bind tcp inline. In Metasploit. Github. https: //github.com/rapid7/metasploit-framework/blob/6.0.37/modules/p ayloads/singles/linux/x64/shell_bind_tcp.rb. Acessado: 29/01/2021.

Sadeghi, A., Aminmansour, F., and Shahriari, H. R. (2015). Tiny jump-oriented programming attack (A class of code reuse attacks). In 2015 12th International Iranian Society of Cryptology Conference on Information Security and Cryptology (ISCISC), pages $52-57$.

Shackelford, E. (2021). TAPing the Stack for Fun and Profit: Shelling Embedded Linux Devices via JTAG. IOActive Labs. https: / / labs. ioactive.com/2021/01/ta ping-stack-for-fun-and-profit.html. Acessado: 05/04/2021.

Spafford, E. H. (1989). The Internet Worm Program: An Analysis. SIGCOMM Comput. Commun. Rev., 19(1):17-57.

Spinellis, D. (2017). A Repository of Unix History and Evolution. Empirical Software Engineering, 22(3):1372-1404.

Symonds, Q. (2020). QS World University Rankings. Top Universities. https : / www . topuniversities.com/university-rankings/world-university-ranki ngs/2020. Acessado: 13/03/2021.

Torvalds, L. (1991). Linux-0.01. In Linux Kernel. Kernel. https://git.kernel . o $\mathrm{rg} / \mathrm{pub} / \mathrm{scm} / \mathrm{linux} / \mathrm{kernel/git/history/history.git/commit/fs/exec}$ . $c$ ?h=0 . 12 \& id=bb 441 db 1 a 90 a 1801 ef 4 e6546417a 8d907c55d92f. Acessado: $05 / 04 / 2021$. 